\title{
STEM Education and Industry Workforce Life-Long Training Platform Development to Faciliate Smart Biopharmaceutical Manufacturing 4.0
}

\author{
Wei Xie \\ Mechanical and Industrial Engineering \\ Northeastern University \\ Boston, U.S. \\ w.xie@northeastern.edu \\ Jinxiang Pei \\ Mechanical and Industrial Engineering \\ Northeastern University \\ Boston, U.S. \\ ji.pei@northeastern.edu
}

\author{
Beverly Kris Jaeger-Helton \\ Mechanical and Industrial Engineering \\ Northeastern University \\ Boston, U.S. \\ b.jaeger@northeastern.edu \\ Hua Zheng \\ Mechanical and Industrial Engineering \\ Northeastern University \\ Boston, U.S. \\ zheng.hua1@northeastern.edu
}

\author{
Jared Auclair \\ Biotechnology and Bioinformatics \\ Northeastern University \\ Boston, U.S. \\ j.auclair@northeastern.edu
}

\begin{abstract}
The biomanufacturing industry is growing rapidly and becoming one of the key drivers of personalized medicine, especially related to cell and gene therapies. However, according to the U.S. Food and Drug Administration (FDA), $80 \%$ of deviations in manufacturing are caused by human error and lack of process knowledge on the manufacturing floor. This not only impacts public health and safety, but also leads to a high risk of failures and financial loss. Traditionally, these manufacturing practitioners have been educated either in Biochemistry or Industrial Engineering (IE), which has led to several key challenges. First, the existing biochemistry education focuses on developing students with science-based knowledge in biology and chemistry; however, this limited foundation lacks basic education in data analytics and biopharmaceutical production process risk management. Second, existing IE approaches do not explore pharmaceutical biotechnology domain knowledge. Thus, driven by the critical needs in the current biopharmaceutical manufacturing industry (biopharma), our multidisciplinary team composed of experts in artificial intelligence (AI), biomanufacturing, regulation, cyberphysical system risk management and automation, biology, and biochemistry guides a multifaceted capstone project. This project focuses on developing an interdisciplinary, modularized and extensible STEM education and industry workforce lifelong training platform. This advanced educational initiative offers integrated AI, bioanalytics, and biomanufacturing process knowledge to yield improved overall quality, system and personnel performance in the pharmaceutical biotechnology sector. By using IE principles grounded in human performance engineering and harnessing the specialized expertise from the biopharmaceutical domain, the interactive STEM Biopharma Training (SBT) program can: (1) be conducted by trainees/learners with various backgrounds, (2) facilitate the systematic development of a world-leading biopharmaceutical manufacturing workforce, (3) minimize biopharmaceutical manufacturing errors and defects, and (4) accelerate biomanufacturing innovation.
\end{abstract}

Keywords-biomanufacturing 4.0, experiential learning, lifelong training, personalized learning, workforce development, multidisciplinary research and education, customized training, capstone

\section{INTRODUCTION}

Industrial cooperative education has been an established and historical strength of Northeastern University's experiential learning brand. For over 100 years, cooperative education (co-op) has been the cornerstone of Northeastern University's educational model. Co-op provides students the opportunity to explore or refine potential career paths, make valuable industry connections, broaden perspectives, and acquire the skills and knowledge needed to succeed. As an enhancement to co-op education at Northeastern University, students are expected to apply science and engineering principles acquired throughout their undergraduate academic and co-op experiences to the design of a product, system, device, and/or process. Students form teams and are assigned their design project and faculty advisor. Projects can be industrially, departmentally, or externally sponsored. In this culminating academic capstone experience, each project includes the development and use of design methodology, formulation of design problem statements and specifications, generation and consideration of alternative solution options, feasibility considerations, and detailed system descriptions.

In Xie et al. [1], the authors propose an integrated framework that unifies research, education/training and industry practice to facilitate knowledge innovation and seamless transition into the skilled workforce, ultimately becoming standard regulations and industry practice. This framework includes an iterative process as shown in Figure 1 below. One pivotal element of this framework is transforming the validated innovative research to education and industry workforce training and ultimately reflecting concept applications in industry practices; see for example [2].

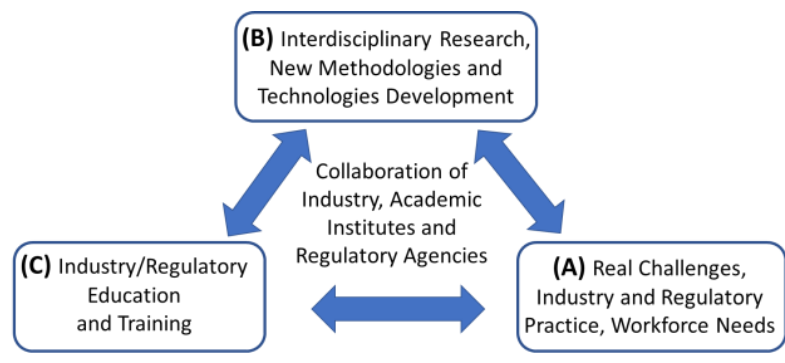

Figure 1. Illustration of integrated research, education/training and industry practice

In the latest industrial revolution, biomanufacturing 4.0, the end-to-end integrated bioprocess becomes the digitalized paradigm, and machines collect process and product data and communicate with other machines via the Industrial Internet of Things (IIoT). Assisted by artificial intelligence (AI), machine learning, and cyber-physical system (CPS) risk management/elimination, machines can use these data to improve processes without human intervention; see [3]. However, traditionally, classroom and lab training and education about biopharma emphasizes bio-science which draws from principles in chemistry and biology, such as Sonia Yakov Cherner et al. [4] and Sparks Wallman et al. [5]. 
There is a critical need for the current students and prospective workforce in biomanufacturing to understand and guide the coherent and optimal decision making for the endto-end integrated biomanufacturing processes. More and more research centers and organizations have begun to offer training in this field. For example, the Biopharmaceutical Analysis Training Laboratory (BATL directed by the author Jared Auclair) at Northeastern University offers internal students and industry practitioners opportunities to keep up with innovations in the biomanufacturing process and AI technology, which will facilitate biomanufacturing 4.0. Similarly, at North Carolina State University, their Biomanufacturing Training and Education Center also offers training in this field.

In terms of emerging AI technology, very limited opportunities for training and education in biomanufacturing are found. While there is a large body of research in the AI and biopharma communities, it is not clear how to effectively translate innovative research to education and training and further to practices. Thus, our goal is to propose a science, technology, engineering and mathematics (STEM) biopharma education and workforce training platform, which unifies research, education/training and industry adoption. We will show the evidence of how the framework functions through integrating the BATL lab training and capstone projects.

\section{TRAINING AND EDUCATION TO ACCELERATE BIOMANUFACTURING INNOVATIONS}

\section{A. Overall Goals}

To facilitate world-leading biomanufacturing workforce development, we provide interdisciplinary multi-level customized education and training, including (1) global industrial/academic training by the Biopharmaceutical Analysis Training Laboratory (BATL) and (2) authors' multidisciplinary undergraduate/graduate classes in biopharmaceutical process development, process data analytics, and industrial \& systems engineering. Authors are guiding and advising a series of capstone projects to transform the frontier research progress into an interdisciplinary education/training curriculum and STEM materials. This program will be adopted by BATL, recognized as an APEC (Asia-Pacific Economic Cooperation) Center of Excellence in Biotherapeutics and Advanced Therapies to provide training throughout an individual's career from high school through advanced training based on both theory and practice in biopharma. In addition, we leverage Boston's location as the top U.S. biopharmaceutical hub, with well over 100 pharmaceutical companies, to collect input from industrial collaborators and facilitate technology transfer.

\section{B. PAT STEM Education Platform Integrating into BATL \\ Training to Accelerate Biomanufacturing Regulation \\ Practice and Industry Innovations}

To facilitate the broad impact and accelerate the biomanufacturing industry and regulation innovations, we develop an iterative STEM education platform described in Section III and further integrate it into the Northeastern Biopharmaceutical Analysis Training Laboratory (BATL). The goal of BATL training is to build skilled human capacity in regulatory sciences to bring safe, effective, and quality medical products to patients and people as quickly as possible. This can improve the biomanufacturing process and reduce the regulatory burden. BATL offers many types of programs including: an APEC-approved course in biotherapeutics and Biosimilars; an ICH trusted-training program in Drug Stability; Intact Mass Analysis, Glycoproteins, \& Quality by Design; and a hands-on graduate course in Protein Mass Spectrometry, to name a few. The existing categories and highlighted new modules that we will develop are shown in Figure 2. As noted, BATL is recognized as an APEC Center of Excellence in Biotherapeutics since March 1, 2017. It has built training relationships with multiple agencies and has been a trusted training partner for the International Council for Harmonisation. At the same time, we have built collaborative training relationships with APEC RHSC Center of Regulatory Excellence in Biotherapeutics and pilot for Advanced Therapies, WHO, and NIIMBL.

The objectives of BATL training include: (i) creating a holistic resource for training and education in the burgeoning field of biomanufacturing, with a novel focus on curricula that integrates manufacturing process analytical technology (PAT) training with real-time analytics training (see [6]); (ii) meeting clear demands in the local biopharmaceutical industry for trained quality control and regulatory experts; (iii) offering workforce development opportunities for biotech and biopharma workers as well as local high school, community college, university, and graduate students; (iv) addressing a global need for convergence and training in regulatory practices related to quality and biologics; and (v) generating opportunities for improvements and innovations in training and procedural efficiencies in biotherapeutics manufacturing, especially related to advanced cell therapies and real-time analytics during the manufacture process.

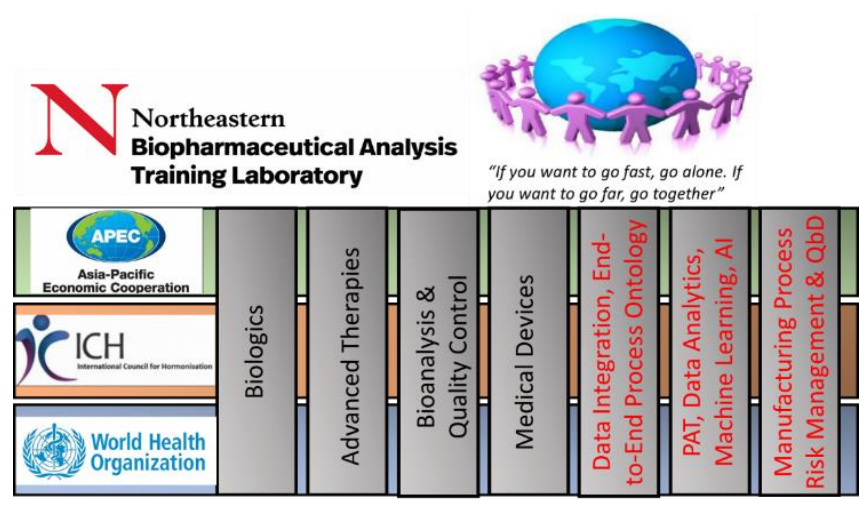

Figure 2. BATL for global biomanufacturing process and analytics training and new AI and process risk management platform facilitating manufacturing innovations

To meet the emerging industrial needs on biomanufacturing 4.0, our multidisciplinary team is transforming the research progress in PAT, including bioprocess modeling, learning, machine learning, risk analysis/management, and AI, to the training and education materials through a series of capstone. Then, we integrate it into BATL training and education; see the highlighted modules in Figure 2. Accordingly, the capstone project design and workforce training are needed to facilitate knowledge transition and help advance the field of biomanufacturing. In Figures 2 and 3, we illustrate how to integrate the new STEM education materials on $\mathrm{AI}$ and machine learning into the BATL existing training program to support the world-leading workforce development and biopharmaceutical production automation. 
Research $\rightarrow$ Training: BATL Research Workflows

(Themes: Analytical, Process, Operations, Standards, Validation)

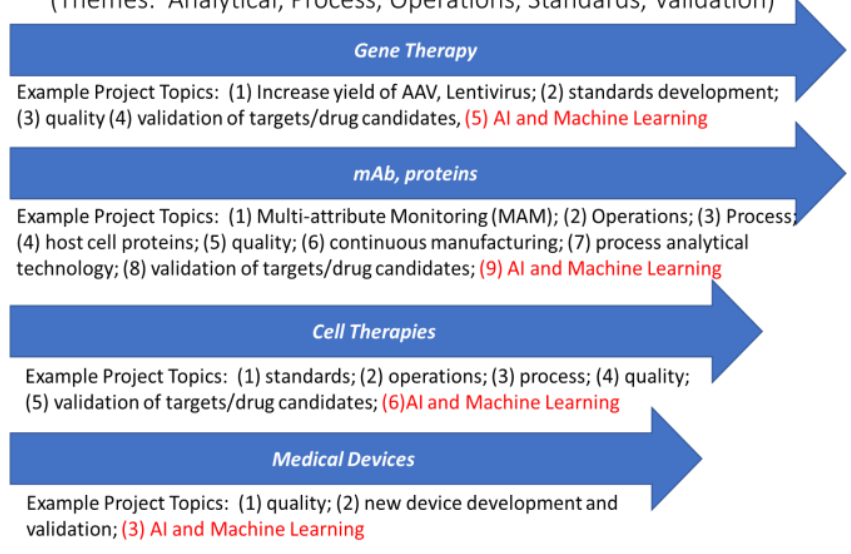

Figure 3. Illustration on integrating the new AI and machine learning into to BATL training program

Experiential Training Methodologies: BATL provides the comprehensive, multi-level experiential learning training throughout an individual's career from high school through advanced training, which starts right from the beginning with theory and practice training. The training is comprised of online training, didactic lectures, chalk talks, case studies, design tree exercises to encourage critical thinking, and handson experimental lab/manufacture work; see Figure 4. Learners have spanned Northeastern undergraduate and graduate students, industry workers, and national and international regulatory professionals.

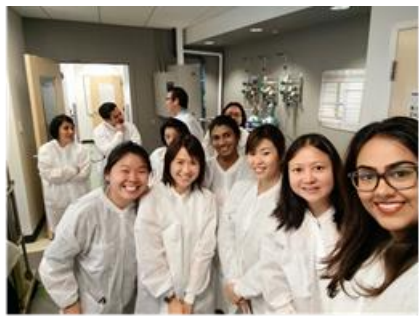

Experiential hands-on learning

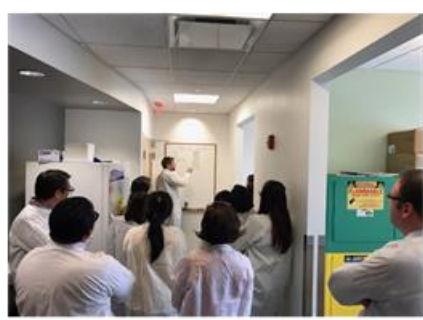

Didactic Lectures and Chalk Talks

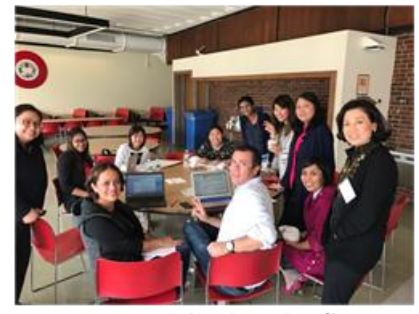

Interactive Case Studies

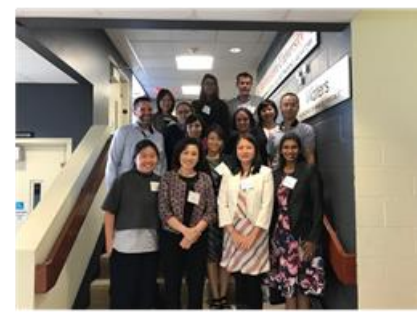

BATL Training Site
Figure 4. Comprehensive, multi-levels experiential education and training at BATL

\section{BATL 1.0: Bioanalysis Training}

The Biopharmaceutical Analysis Training Laboratory (BATL) at Northeastern University was established in 2014 in response to the critical needs from biopharmaceutical manufacturing industry and regulation. It provides deeper training in sophisticated bioanalysis. BATL has had 625 trainees between March 2017 and October 2018 with a gradual ramp-up in the first few years -from 2014 through March 2017 there were 138 students trained.

We have gained momentum since 2017 with 426 global regulators, 124 industry employees, and 75 Northeastern University (NU) students trained through this program. Its first-generation facilities include the Mass Spectroscopy laboratory, protein characterization bays, and a 50-seat classroom of $\sim 4,000$ square feet. This facility offers experiential learning, training in both biochemical and biopharmaceutical analysis theory and practical methodologies; see the examples in Figures 3 and 4.

\section{BATL 2.0: Bioanalysis + Biomanufacturing Training}

Then, BATL links biomanufacturing to corresponding analysis methods and provide theoretical and experiential learning opportunities. In addition to standard bioanalysis training, we teach foundational principles that empower workers to better understand optimal batch conditions and give workers judgment skills and tools to innovate a particular process or to know when to halt a batch. This foundation creates a training experience that recapitulates commercial conditions and prepares for a large pipeline of trainees. It focuses on the following key areas as shown in Figure 5, including (1) cell-based therapies; (2) personalized medicines; and (3) continuous analytics - linking analysis and biomanufacturing.

To meet the industry needs, BATL has a continuously increasing training scale as described below.

- Our aspiration is to bring our number of trainee participants to 1000 per year as quickly as possible post-expansion -by the year 2021. In Table 1, we predict how our trainee demographics will stratify, once we reach that level.

- Our initial projection of the number of industry employees in the right-hand column in Table 1 is conservative; and we expect this to continue growing in proportion to the baseline, in particular due to the biomanufacturing component.

- The current composition of active trainees is 625, comprising 426 global regulators, 124 industry employees, and 75 Northeastern university students

Table 1. The background distribution of trainee demographics

\begin{tabular}{|c|c|c|c|c|}
\hline $\begin{array}{c}\text { Trainee } \\
\text { Education Level }\end{array}$ & $\begin{array}{c}\text { Trainee Professional Goals } \\
\text { (biomanufacturing \& } \\
\text { regulatory) }\end{array}$ & $\begin{array}{c}\text { Projected } \\
\text { proportion of } \\
\text { overall } \\
\text { trainees }\end{array}$ & $\begin{array}{c}\text { Projected \# of } \\
\text { overall } \\
\text { trainees } \\
\text { (based on } \\
1000 \text { per year) }\end{array}$ & $\begin{array}{c}\text { Projected \# of industry } \\
\text { employed trainees- } \\
\text { upskilling } \\
\text { (biomanufacturing only, } \\
\text { not regulatory) }\end{array}$ \\
\hline High school & Entry level employment & $5 \%$ & 50 & 0 \\
\hline Associate & $\begin{array}{c}\text { Entry level employment, } \\
\text { Upskilling, }\end{array}$ & $5 \%$ & 50 & 75 \\
\hline Bachelors & $\begin{array}{c}\text { Entry level employment, } \\
\text { Upskilling, }\end{array}$ & $70 \%$ & 700 & 150 \\
\hline Graduate & $\begin{array}{c}\text { Mid level employment, } \\
\text { Upskilling, Certificate }\end{array}$ & $20 \%$ & 200 & 100 \\
\hline
\end{tabular}




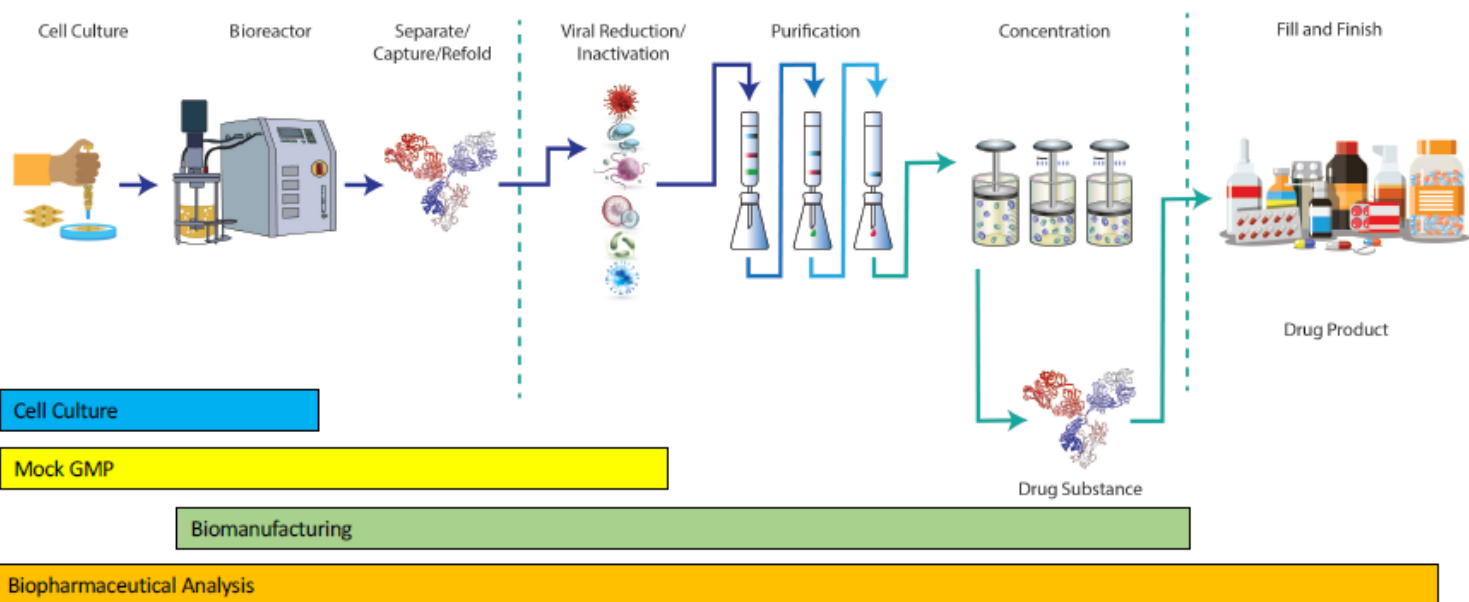

Figure 5. Bioanalysis + Biomanufacturing Training programs in BATL 2.0

\section{BATL Training Equipment for "Bioanalysis + Biomanufacturing + $\mathrm{Al}^{\prime}$}

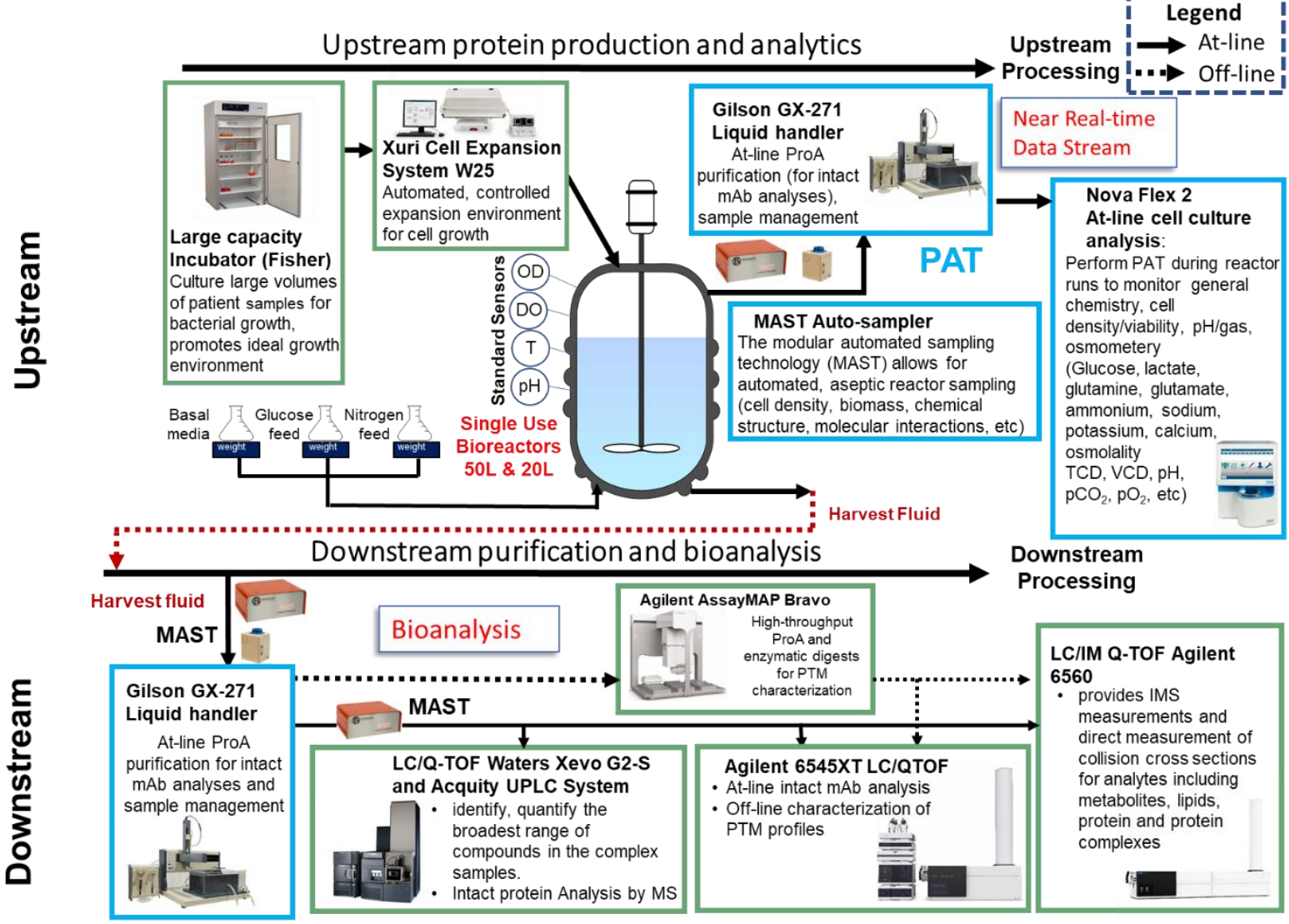

Figure 6. Integrated biomanufacturing process and monitoring facilities at BATL facilitating the in-laboratory experiential and hands-on training. 


\section{BATL 3.0: Bioanalysis + Biomanufacturing + AI Training}

The critical needs in biomanufacturing 4.0 and end-to-end bioprocess automation call for big data analytics, interpretable AI, and advanced Process Analytical Technologies (PAT). Based on the authors' innovative research in building a datadriven decision support tool, an integrated training and education platform is being developed to accelerate the development of modular, flexible, reliable, intensified, automated, and continuous biomanufacturing. At the same time, the PAT platform and decision support tool will be validated by using historical process data and continuous/batch-based biomanufacturing systems. Our PAT can leverage existing mechanistic models and facilitate learning end-to-end bioprocess biological/physical/chemical interactions and dynamics at molecular, cellular, and system levels.

Under the funding support from the Massachusetts Life Sciences Center (MLSC) for advanced biomanufacturing workforce development, the Biopharmaceutical Analysis Training Laboratory is poised to leverage current and prospective training resources as shown in Figure 6 to provide hands-on training and education services on bioanalysis, integrated biomanufacturing process, and AI. The highlighted PAT sensor network and processing facilities in Figures 5 and 6, such as MAST auto-sampler and Nova Flex 2, can facilitate the comprehensive data collection, process analytics, and machine learning.

\section{INTERACTIVE BIOPHARMA TRAINING AND LIFE-LONG LEARNING PLATFORM DEVELOPMENT}

Biopharmaceutical production faces critical challenges, including process complexity, high variability, and long lead times; see [7]. Existing mechanistic models have been developed for individual biomanufacturing unit operations and associated transport phenomena. They are modeled by ordinary or partial differential equations (ODE/PDE). With new trends, operations research/management (OR/OM) methods for system analytics and decision making for biomanufacturing plants include mixed integer linear programming, supply chain management methodologies and decision-support tool and queuing network theory; these have been used to analyze resource planning, scheduling, and material consumption costs, without including detailed biological/physical/chemical phenomena. Overall, existing methodologies have several key limitations: (1) mechanistic models are usually deterministic, yet real bioprocess operations are stochastic; (2) OR/OM approaches focus on developing general methodologies without incorporating the mechanistic information, which limits their performance, interpretability, and adoption; and (3) existing mechanistic studies focus on individual unit operations without providing a plantwide framework for reliable and interpretable end-toend risk analysis and control.
To overcome the critical challenges -both current and emerging- in the biomanufacturing industry and the limitations of existing approaches, we propose a new biopharmaceutical production Process Analytical Technologies (PAT) platform that addresses critical needs in biomanufacturing automation and facilitate biomanufacturing 4.0; see for example [2]. In the first stage of development, this platform provides ontology-based bioprocess data integration to connect distributed/multi-scale/heterogeneous data through causal relationships and dynamic interactions. An end-to-end bioprocess hybrid model characterizes the spatial-temporal interdependencies of critical process parameters and product quality attributes, provides risk- and science-based production process understanding, and supports the coherent and optimal decision making. The approach can leverage existing mechanistic models, and facilitate the learning of end-to-end bioprocess biological/physical/chemical interactions and dynamics at molecular, cellular, and system levels. Thus, the education modules developed based on the first-stage PAT platform development include data integration, end-to-end bioprocess ontology graphing, as well as data and process analytics; see the highlights in Figure 2.

The authors are guiding the series of capstone design projects to seamlessly transform the validated research progress to education/training and we are developing an AI, machine learning, and PAT-based interactive STEM Biopharma Training Platform to accelerate biomanufacturing workforce innovations. By utilizing the IE principles grounded in process flow expertise and human performance engineering, and harnessing the domain-specific expertise from the biopharmaceutical realm, we present a specialized education platform design to facilitate an efficient and fast learning/training experience. We also present the outcome from the first capstone project, which will be integrated into BATL training to enlarge the broad impact and accelerate the local and global biomanufacturing workforce innovations.

\section{A. Background on Our Capstone Project Team}

In our capstone project, the advisor team (see Figure 7) consists of experts in: (1) interpretable AI, big data analytics, process analytics, design of experiments, computer simulation, cyber-physical system learning, risk management, and automation for biomanufacturing and supply chain; (2) biotechnology, regulation and drug quality; (3) biomechanics and mechanobiology; and (4) process mapping, human factors \& human performance engineering, and engineering education. At the same time, the advisor team has over ten years' academic and industry experiences in manufacturing and supply chain analytics and management, as well as biopharmaceutical analysis and production regulation. The student teams include senior undergraduate students majoring in industrial engineering. They have all completed multiple co-ops and bring a wealth of experience to this valuable, implementable, and measurable capstone design project . 
Students: Brian Bae, Rolando Cubillos, Laura Hobbs, Carter Rose, Minas Zahopoulos
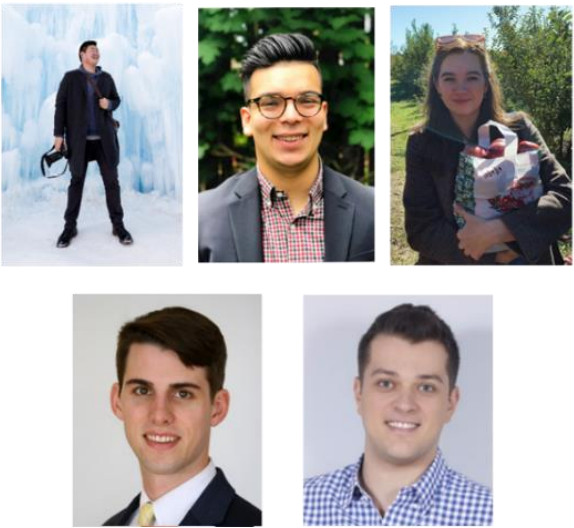

Multidisciplinary Advisors: Beverly-Kris Jaeger Helton, Paul Pei, Wei Xie, Jared Auclair, Hua Zheng
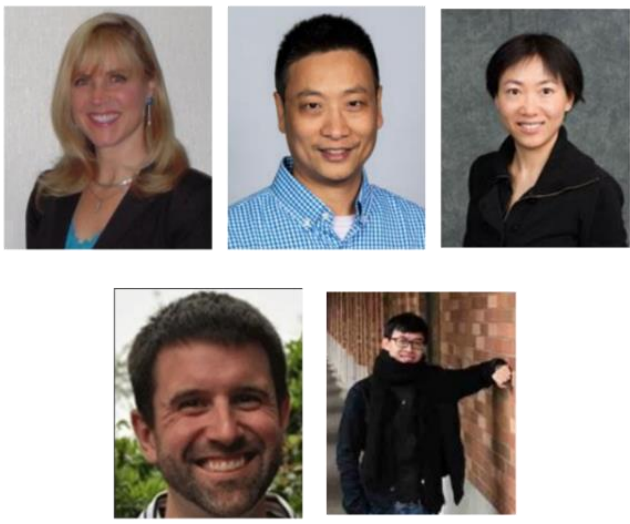

Figure 7. Our capstone project team with multidisciplinary advisors

\section{B. Education and Training Methodologies and Outcome} from Capstone Project

To facilitate rich and informed experiential learning, the education methodologies in our interactive STEM Biopharma Training Platform include: (1) Module-based human-centered learning and training, see for example Figure 8; (2) Integrated and multifaceted education through BATL; (3) On-site visits; (4) Survey of industrial and academic leaders to collect the recent needs from industry; and (5) Learning assessment and quizzes (pre- and post-). In designing the capstone project, we offer a pre-quiz for trainees to test their prior knowledge about biomanufacturing processes and assess their analytical skills. In the training and learning protocol, interactive quizzes are offered in the middle for trainees to self-evaluate their learning progress. The summary quizzes are used to assess knowledge retention and training quality. Based on the results of all the quizzes, a certificate of successful completion of the training modules is issued. In addition, quiz and survey data serve to guide further improvement on the STEM Biopharma Training Platform.

The design methodology of this training platform is intentional; it is modular-based, research-driven, and humancentered learning and training. The modularized design can support customized life-long education, and it is also extensible so that we can easily incorporate the recent research progress and new developed education materials into our STEM biopharma training platform. In the first stage of development, this training and education platform we design covers business processes in biomanufacturing and advanced data/process analytics together with knowledge transition and assimilation in current and prospect workforce.

The outcome from this capstone project includes three modules as shown in Figure 8. To assist Biomanufacturing 4.0, in Module 1, we present learners an overview of the endto-end biomanufacturing process, including upstream bioreaction and downstream purification. We also provide the insights as to how various factors (e.g., critical process parameters, critical quality attributes, and any controllable factors) introduced in different stages of the bioprocess interact with each other, and eventually impact the production outputs, such as product quality, production cycle time, productivity, and yield. Apart from traditional bioanalysis training that is biology and chemistry based, we offer trainees a big picture view and understanding of integrated biomanufacturing process. It has been shown extremely helpful for prospective workforce to have an end-to-end view. At the same time, it provides foundation for advanced data and process analytics with interpretable AI that explores the causal relationships across unit operations and hundreds of parameters and quality attributes.

In Module 2, trainees are exposed to current and recently developed bioprocess modeling and AI methodologies in both the overall biomanufacturing process and each individual unit operation. Fundamentally, the methodologies can be divided into first-principle models, statistical models, and the hybrid model. We review the commonly used first-principle and statistical models and analytics approaches, including PDE/ODE based first-principle models for each operation unit and regression analysis, which explores the correlation among quality attributes, such as impurity, protein level, media formulation and critical process parameters, e.g., temperature, $\mathrm{pH}$, dissolved oxygen, and others. Built on our research progress, we further present the bioprocess ontology-based relational graphical models connecting distributed/multiscale/heterogeneous data through causal relationships and dynamic interactions within and between various operation units. Then, we build the end-to-end production process probabilistic knowledge graph to support the risk-based and science-based understanding of the bioproces and modeling of CPPs/CQAs spatial-temporal interdependencies. It is a hybrid model integrating information from first-principle models from each operation unit and data. The domain knowledge presented in Module 1 is leveraged to create a bioprocess knowledge graph that provides foundation of the probability models.

In Module 3, we utilize the case studies to compare the performance from different bioprocess modeling and analytics approaches and drive insights home for trainees. For example, we explore contribution of dissolved oxygen level and gene stability to producing impurities in the cell culture unit operations and further dependence of its contribution on other parameters such as $\mathrm{pH}$. We sharpen regression analysis by incorporating multi-tier regressions (dynamic regression) where the output in one stage becomes input to the next-stage regression. The advanced regression analyses explore both within- and across- unit operation relationships. Using regression as a benchmark, we move forward with analyzing causality across multiple unit operations using conditional 
probability models. We compare with regression analysis and gain deeper insights into the graphical model by exploring wholistic causality.

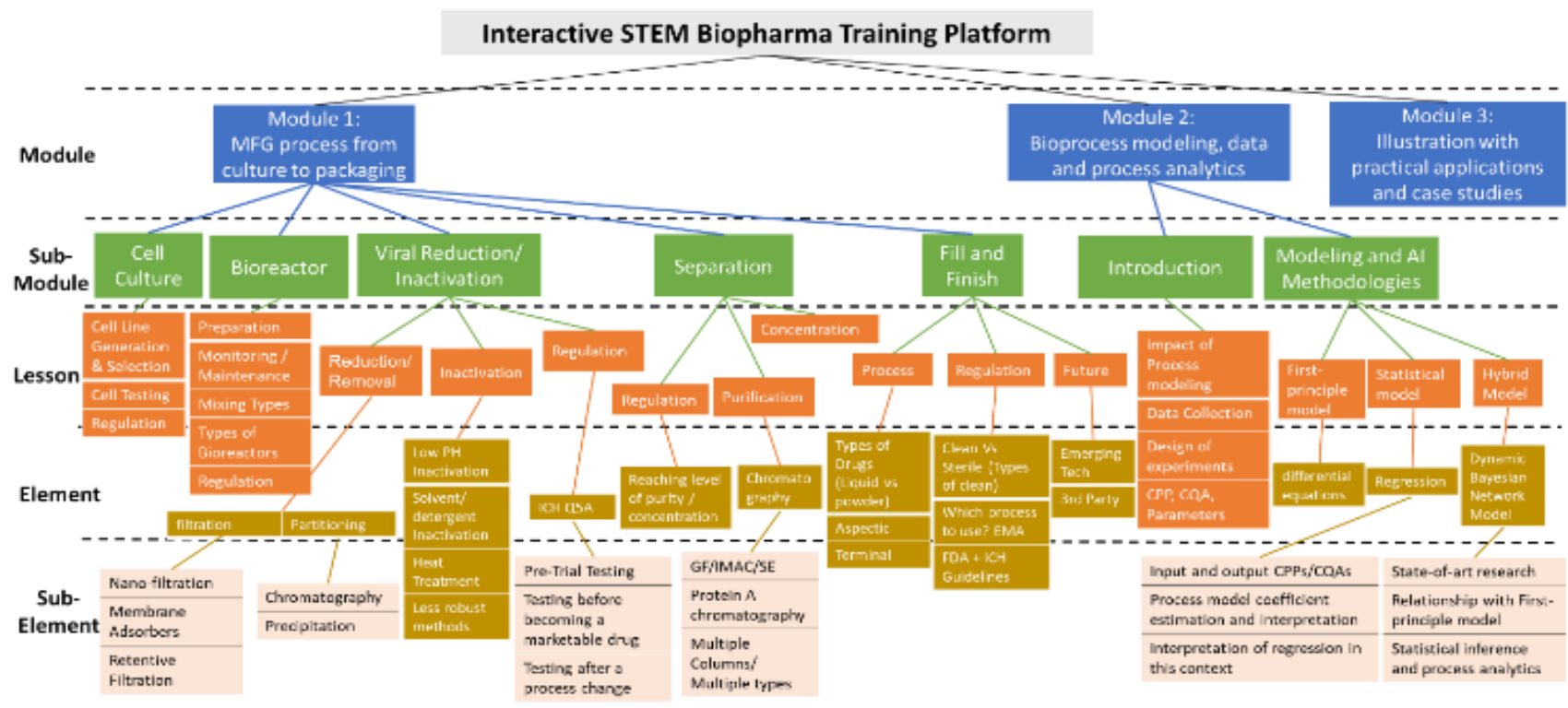

Figure 8. Concept map of interactive STEM Biopharma Training Platform from the first capstone project
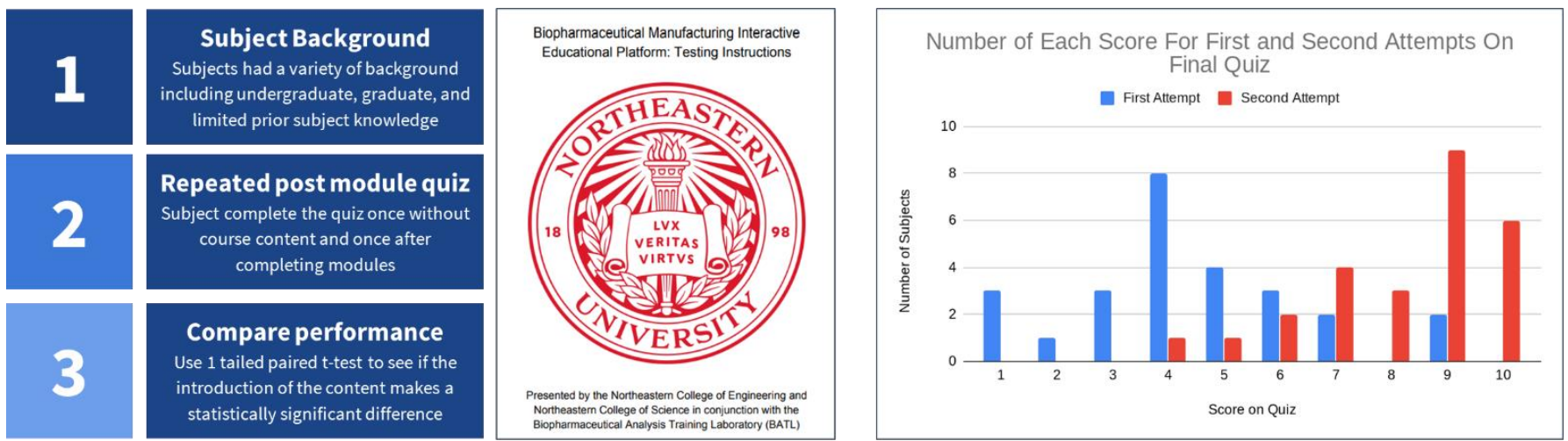

Figure 9. Testing methods and analyses of the developed interactive training and education platform.

Capstone Project Plan and Management: This twosemester capstone project started in September 2019 and concluded at the end of April 2020. In the first semester, the student team focused on background learning and training; and compact video lectures were used to ground every team member on the same page. At the same time, the student team mastered advanced statistics and IE methodologies as shown below. Innovative research in interpretable AI and advanced data analytics has been introduced in the student team. When the team successfully built the basic knowledge, a concept map as shown in Figure 8 was constructed to connect thoughts on module design and project flow. Then, the team integrated the content to a web host so the platform could be maximally leveraged. At the end of the project, the final product was an award-winning integrated web-host training and education platform. In this program, trainees take a pre-quiz before starting the training. Then a sequence of online interactive training modules consisting of illustrated video and text lectures are followed by self-evaluation quizzes will be given. In particular, advanced data analytics and AI modules are provided to keep pace with current innovative research in the biomanufacturing field. Basic data techniques and skills are reviewed. Case studies are provided and insights are driven from the training and learning process. Upon successful completion of the training modules, certificates are issued.

STEM Online Education Platform Performance Evaluation: Based on the collected data from participants, we conducted the one-tailed paired $t$-test to assess the performance of our preliminary education platform. Since the obtained P-value of 0.00000 is less than the $5 \% \alpha$ confidence level, the learners show a statistically significant improvement in their scores after completing the online modules. The data illustrated in Figure 9 also show the significant increase in test scores between the two attempts.

Integration with BATL to Improve the Broad Impact: The STEM training platform will be integrated with current workforce training practice offered at the BATL site. The new emphasis is on building AI and advanced data analytics in the training and education curriculum. The project team visited manufacturing facilities on-site and connected learning modules with physical equipment and systems. These visits 
reinforced the learning and training process. We plan to visit more facilities to offer trainees opportunities to gain a better grasp on more aspects of the complex and essential biomanufacturing processes. In the design phase, we invited volunteers to participate and validate the training and learning modules. Based on their feedback, we incorporated updates in module content and quizzes resulting in a valid and effective training platform for biopharmaceutical production.

\section{CONCLUSIONS}

Driven by the critical industry needs and next-generation Biomanufacturing 4.0, our multidisciplinary advisors guided a capstone project developing an interactive, interdisciplinary, modularized and extensible STEM education and industry workforce life-long training platform. It covers data integration and analytics, end-to-end production process modeling and the associated analytics, machine learning, and AI. It provides the comprehensive background knowledge of biopharmaceutical production processes and reviews the existing bioprocess mechanisms and commonly used PAT methodologies, including the first-principle approaches and statistical approaches. In the capstone design project, we transformed the recent research progress on the end-to-end bioprocess hybrid modeling and analytics into educational materials. We will further integrate this STEM platform into BATL training and accelerate biomanufacturing workforce innovations. To facilitate exponential progressive learning, various methodologies -including online training, didactic lectures, chalk talks, case studies, design-tree exercises to encourage critical thinking, and hands-on experimental lab/manufacture work - to facilitate a successful and valuable interactive learning experience. The automatic online quiz allows us to collect feedback on the performance of our STEM education platform, which can guide us to iteratively improve the training and inspire new research development. The preliminary study results indicate that this platform can improve the learners' knowledge to support biomanufacturing 4.0 industry innovation.

\section{ACKNOWLEDGMENTS}

We acknowledge all the efforts and passion from the capstone students, including Brian Bae, Rolando Cubillos, Laura Hobbs, Carter Rose, and Minas Zahopoulos, who formed a strong foundation for success in the development of the interactive STEM Biopharma Training (SBT) program and platform. We also appreciate the great suggestions and feedback from other capstone teams' students and advisors.

\section{REFERENCES}

[1] Wei Xie, Jared Auclair, and Jinxiang Pei. "An Integrated Research, Education/Training, and Industry Practice Framework to Accelerate the Innovation in Biopharmaceuticals Manufacturing and Eliminate Drug Shortage". 2019 ASEE Zone I Conference \& Workshop, Niagara Falls, NY, 2019, April. ASEE Conferences, 2019. https://peer.asee.org/33775 Internet. 26 Aug, 2020.

[2] Wei Xie, Bo Wang, Cheng Li, Dongming, Jared Auclair, "Interpretable Biomanufacturing Process Risk and Sensitivity Analyses for Qualityby-Design and Stability Control”. https://arxiv.org/abs/1909.04261.

[3] http://www.biopharminternational.com/industry-40biopharmaceutical-manufacturing-0? pageID $=1$.

[4] Yakov Cherner, Maija Kuklja, and Alexander Rudy. "Customizable Virtual X-Ray Laboratory: An Innovative Tool for Interactive Online Teaching and Learning". 2014 ASEE Annual Conference \&
Exposition, Indianapolis, Indiana, 2014, June. ASEE Conferences, 2014. https://peer.asee.org/20242 Internet. 26 Aug, 2020

[5] Yakov Cherner, Sonia Sparks Wallman, Margaret Bryans, and Marina Taranova. "Virtual and Blended Liquid Chromatography Laboratories for Chemical and Biological Engineering Education". 2011 ASEE Annual Conference \& Exposition, Vancouver, BC, 2011, June. ASEE Conferences, 2011. https://peer.asee.org/18361 Internet. 26 Aug, 2020

[6] Cynthia A. Challener, "Improving PAT for Biologics," BioPharm International, DOI (2017).

[7] K. Dalgaard, W. C. Garstner, From Science to Operations: Questions, Choices, and Strategies for Success in Biopharma, McKinsey \& Company, DOI (2014). 